\title{
The Influence Mechanism of Proactive Personality on Innovation Behavior of Military Civil Personnel
}

\author{
Yanhui Tian ${ }^{1, *}$ Ting Wei ${ }^{1}$ Zhengyin Wang ${ }^{1}$ Fei Liu ${ }^{1}$ Hui Liu ${ }^{1}$ \\ ${ }^{I}$ Communication of Information and Communication, National University of Defense Technology, Xi'an, \\ Shaanxi 710106, China \\ "Corresponding author. Email: tianyanhui05@163.com
}

\begin{abstract}
With the progress of reform, more and more outstanding local talents have joined the civil personnel teams and have become an important part of military personnel. How to give full play to their intelligence and inspire innovative behaviors has become an important issue for military human resource management. This study uses the opportunity of centralized training for civil personnel in the military to investigate 450 civilians using the Proactive Personality Scale, Occupational Resilience Scale, and Innovative Behavior Scale to explore the influencing factors and mechanisms of civilians' innovative behavior. A total of 419 valid questionnaires were collected. The results of correlation analysis showed that proactive personality was significantly positively correlated with professional toughness $(\mathrm{r}=0.56, \mathrm{P}<0.05)$ and innovative behavior $(\mathrm{r}=0.58, \mathrm{P}<0.05)$; professional toughness was significantly positively correlated with innovative behavior $(r=0.60, \mathrm{P}<0.05)$. The PROCESS3.2 macro program developed by Hayes was used to test the mediating effect of career resilience between proactive personality and innovative behavior, and bootstrap $=5000$. The results show that the mediating effect of career resilience was 0.245 , and the $95 \%$ confidence interval was $[0.173,0.323]$. The direct effect of proactive personality is 0.387 , and the $95 \%$ confidence interval is [0.293, 0.480], which proves that professional resilience plays a part of mediating role between the proactive personality and innovative behavior of military civilians. This conclusion also has certain enlightening significance for the management and use of military civilian personnel. Inspiring their innovative behaviors can start with cultivating proactive personality and professional resilience, both of which work together to play a role.
\end{abstract}

Keywords: Proactive personality, Innovative behavior, Professional resilience, Military civil personnel.

\section{INTRODUCTION}

With the continuous advancement of the reform of the military human resources system, more and more outstanding social talents have joined the military civilian teams, playing an increasingly important role in national defense and army building. According to public reports, starting from the military's first public recruitment of civilian personnel in 2018, 9,297 people were recruited that year, 19,523 people were recruited in 2019 , and 27,073 people were recruited in 2020 , with a trend

*Project: The phased results of the National Social Science Fund project "Research on the Ideological and Political Quality of Military Civilians in the New Era (19XGL028") and

"Research on the Professional Spirit of Military Civilians in the New Era (20BGL312)". of increasing year by year. It can be predicted that more and more civilian personnel will enter military camps and become an important part of military human resources. How to manage and make good use of civil personnel, make them adapt to military camp life as soon as possible, and make good use of the unique advantages of civil personnel has gradually become the focus and hot topic of military human resource management departments.

From the perspective of the recruitment conditions for civil personnel, most positions require candidates to have a bachelor's degree or above, and some positions require master's or even doctoral candidates. In terms of the use of civil personnel, the vast majority of civil personnel are 
assigned to technical positions and engage in work closely related to the major they are studying. They are basically able to achieve professional counterparts and make the best use of their talents. For civil personnel, their greatest advantage and distinctive feature is their strong innovation ability and high work vitality. These qualities are also the core qualities that employers value most. Therefore, stimulating the creativity of civil personnel and enabling them to inject new momentum into army building and development has increasingly become a practical problem that urgently needs to be resolved in the process of civil personnel management. Innovative behavior refers to the process by which an individual generates or implements new ideas to improve performance or solve work-related problems [1]. It is the external manifestation of employees' internal creativity [2], which plays a vital role in organizational innovation and maintaining competitiveness. [3] Innovative behavior is particularly important among highly educated and knowledgeable employees, and it is also a hotspot of academic research in recent years. Existing studies have shown that the factors that affect innovation behavior can be roughly summarized into three categories: The first is organizational factors, such as inclusive leadership in the leadership type [4]. The authorization behaviors in leadership behaviors will all have an impact on individual innovation behavior; The second is personal factors, such as individual psychological capital [6], self-monitoring personality [7], etc., which will have a certain impact on innovative behavior; The third is the impact of contextual factors, such as work-family relationships, and China's unique cultural environment [8] on innovative behavior. Among the many influencing factors, personal factors are undoubtedly the most fundamental and basic nearend variables, which play a decisive role in the innovation behavior of individuals. Therefore, this research takes the proactive personality of the civil personnel as a starting point, discusses its influence on innovation behavior and its internal mechanism, and tries to reveal the "black box" that affects the innovation behavior of civil personnel.

With the rise of positive psychology thoughts, active personality related to individual positive psychological traits has increasingly attracted the attention of academic circles and has become a new research perspective [9]. Proactive personality refers to the tendency to positively influence the surrounding environment, be good at discovering opportunities, and work towards goals with practical actions [10]. Innovative behaviors, as informal regulations and work behaviors that are difficult to quantify in the organization, are easily affected by individual proactive personality. First, the proactive personality has the characteristics of being good at discovering opportunities, being free from environmental constraints, and daring to meet challenges, which can encourage individuals to solve problems creatively; second, individuals with proactive personality will invest more in knowledge sharing activities, increase their own knowledge accumulation, and provide necessary knowledge reserves for innovative behaviors [9]. Therefore, this study predicts that the proactive personality of civil personnel will have a positive impact on their innovative behavior. In addition, studies have shown that individuals with proactive personality are better at integrating and utilizing various social resources in the process of interpersonal communication, constructing a high-quality social support network, and are more likely to recover from suffering temporary losses when faced with setbacks in the workplace, showing strong professional resilience. As an organizational behavior with a high failure rate, innovative behavior needs strong professional resilience as support. First of all, as an internal ability to deal with external challenges and setbacks, professional resilience can be seen as a protective factor for individuals in the face of innovation failure, effectively protecting the individual's innovation motivation; Secondly, research also shows that individuals with high professional resilience have a stronger spirit of exploration and innovation, and when faced with challenges brought about by innovation, they are more likely to adjust themselves to the best working conditions and produce higher innovation performance [11]. Based on the above analysis, this study believes that professional resilience of civil personnel plays an intermediary effect in the process of the proactive personality of civilians influencing innovative behaviors, and transmits the influence of proactive personality on innovative behaviors. The research model is shown in "Figure 1". 


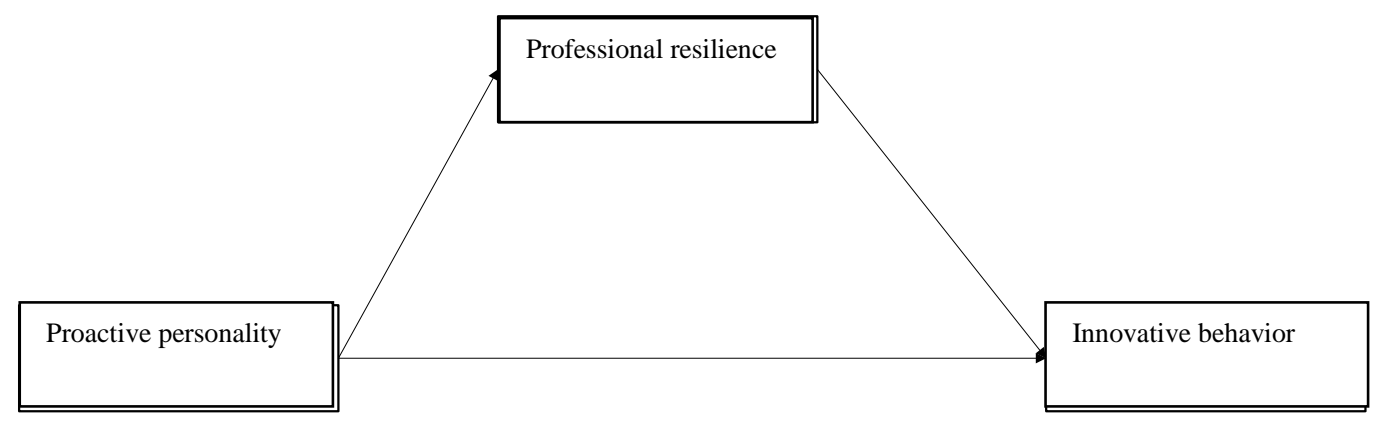

Figure 1 The influence mechanism of proactive personality on innovative behavior.

\section{RESEARCH METHODS}

\subsection{Research Objects}

Taking advantage of the opportunity for centralized training of newly recruited civilian personnel in the military, using convenient sampling methods, 450 questionnaires were distributed from 10 civilian personnel training courses in Xi'an, Wuhan, Nanjing and other places, and 426 valid questionnaires were recovered, with an effective recovery rate of $94.7 \%$. Among the survey respondents, 381 were males, accounting for $89.4 \%$, and 45 were females, accounting for $10.6 \%$; 80 were married, accounting for $18.8 \%$, and 346 were unmarried, accounting for $81.2 \%$; 391 are undergraduates, accounting for $91.8 \%$, and 35 are graduates, accounting for $8.2 \%$. Among the respondents, the lowest age is 21 years old and the oldest is 36 years old, with an average of 26.1 years and a standard deviation of 3.03.

\subsection{Research Tools}

\subsubsection{Proactive Personality Questionnaire}

The research used the proactive personality questionnaire compiled by Bateman and Crant [12]. This questionnaire is also a common questionnaire for measuring individual proactive personality, and it is currently the most authoritative and widely used measurement tool. The questionnaire has a single-dimensional structure, with a total of 10 items, and uses Likert 5-point scoring. "1-5" respectively indicate "completely inconsistent to completely consistent". The higher the score, the more obvious the proactive personality traits. In this study, its Cronbach's $\alpha$ coefficient is 0.799 .

\subsubsection{Professional Resilience Scale}

The research used the occupational resilience subscale [13] in the occupational motivation scale developed by London, a single dimension, a total of 5 items, and used Likert 5-point scoring, 1-5 (completely inconsistent to completely in line). The higher the score, the stronger the professional resilience. The Cronbach's $\alpha$ coefficient for internal consistency in this study is 0.782 .

\subsubsection{Innovative Behavior Questionnaire}

Using the innovation behavior scale compiled by Scott and Bruce [14], the scale has a total of 6 items, a single-dimensional structure, and a Likert5 point scoring. "1-5" means "completely inconsistent to completely consistent". The total scores of all items are divided by the number of items. The higher the score, the stronger the innovative behavior. In this study, its Cronbach's $\alpha$ coefficient is 0.888 .

\subsubsection{Control Variables}

In order to prevent the demographic variables of the survey subjects from interfering with the relationship between the main research variables, this study uses gender, age, educational background, and marriage status in the demographic variables as additional variables to control the data statistics.

\subsection{Data Processing}

The validity of the returned questionnaire is judged and data is entered, and the data is processed by reliability analysis, correlation analysis, regression analysis and other methods. The statistical software used is SPSS22.0 and PROCESS3.2 macro program developed by Hayes. 


\section{RESEARCH RESULTS}

\subsection{Common Method Deviation Test}

The data used in this study all come from the self-evaluation of the survey respondents, and will inevitably be affected by common method deviations. Therefore, the researchers test the possible methodological effects. Using the Harman single-factor method for reference from the previous research, the exploratory factor analysis of all 21 items involved in the questionnaire was carried out. The results of the unrotated factor analysis showed that a total of 4 common factors with characteristic roots greater than 1 can be extracted. Among them, the first common factor can explain $35.9 \%$ of the common variance variation, which is lower than the critical standard of $40 \%$, indicating that there is no serious common method deviation in this study.

\subsection{Descriptive Statistics and Correlation Analysis of Variables}

The mean, standard deviation and correlation analysis of the variables are shown in "Table 1". The correlation between demographic variables and the main research variables has not reached the significant level, and there is a significant correlation between the main research variables. Among them, proactive personality is significantly positively correlated with professional resilience $(\mathrm{r}=0.56, \quad \mathrm{p}<0.01)$, and significantly positively correlated with innovative behavior $(\mathrm{r}=0.58$, $\mathrm{p}<0.01$ ); professional resilience is significantly positively correlated with innovative behavior $(r=0.60, p<0.01)$, the correlation between these variables provides a basis for the hypothesis testing of subsequent research.

Table 1. Descriptive statistics and correlation analysis of variables

\begin{tabular}{|l|l|l|l|l|l|l|l|l|l|}
\hline Variables & $\mathrm{M}$ & $\mathrm{SD}$ & 1 & 2 & 3 & 4 & 5 & 6 \\
\hline 1 Gender & 1.11 & 0.31 & 1 & & & & \\
\hline 2 Education background & 2.08 & 0.27 & $0.23^{* *}$ & 1 & & & & \\
\hline 3 Marriage situation & 1.81 & 0.39 & $-0.19^{* *}$ & $-0.12^{*}$ & 1 & & & \\
\hline 4 Age & 26.08 & 3.03 & $0.18^{* *}$ & $0.21^{* *}$ & $-0.55^{* *}$ & 1 & & \\
\hline 5 Proactive personality & 3.72 & 0.54 & -0.03 & 0.04 & 0.02 & -0.06 & 1 & \\
\hline 6 Professional resilience & 4.21 & 0.55 & 0.06 & 0.07 & -0.02 & 0.04 & $0.56^{* *}$ & 1 \\
\hline 7 Innovative behavior & 4.04 & 0.58 & -0.01 & 0.04 & -0.02 & -0.03 & $0.58^{* *}$ & $0.60^{* *}$ & 1 \\
\hline
\end{tabular}

\subsection{Test of the Mediating Effect of Professional Resilience}

In order to avoid the pollution of the research results caused by the non-normality of the data distribution, this study uses the PROCESS3.2 macro program written by Hayes [15] to test the mediating effect of professional resilience between proactive personality and innovative behavior. The researchers select Model 4, adopt the deviationcorrected non-parametric percentile Bootstrap method, set Bootstrap $=5000$, and control demographic variables such as gender and educational background of the civil personnel. The results are shown in "Table 2" and "Figure 2".
Proactive personality has a significant positive predictive effect on innovation behavior $(\beta=0.62$, $\mathrm{p}<0.001)$; Incorporating the mediating role of career toughness, proactive personality has a significant positive impact on professional resilience $(\beta=0.58, p<0.001)$, and the positive impact of professional resilience on innovation behavior also reaches a significant level $(\beta=0.44$, $p<0.001)$, the direct effect of proactive personality on professional resilience is still significant $(\beta=0.37, p<0.001)$; This proves that professional resilience plays a part of the mediating role between the proactive personality and innovative behavior of civil personnel, and the research hypothesis has been confirmed.

Table 2. Bootstrap test of the mediating effect of professional resilience

\begin{tabular}{|l|l|l|l|l|}
\hline & Effect & Boot SE & BootLLCl & BootULCl \\
\hline Direct effect & 0.36 & 0.48 & 0.27 & 0.46 \\
\hline Indirect effect & 0.26 & 0.39 & 0.18 & 0.33 \\
\hline Gross effect & 0.62 & 0.437 & 0.54 & 0.71 \\
\hline
\end{tabular}




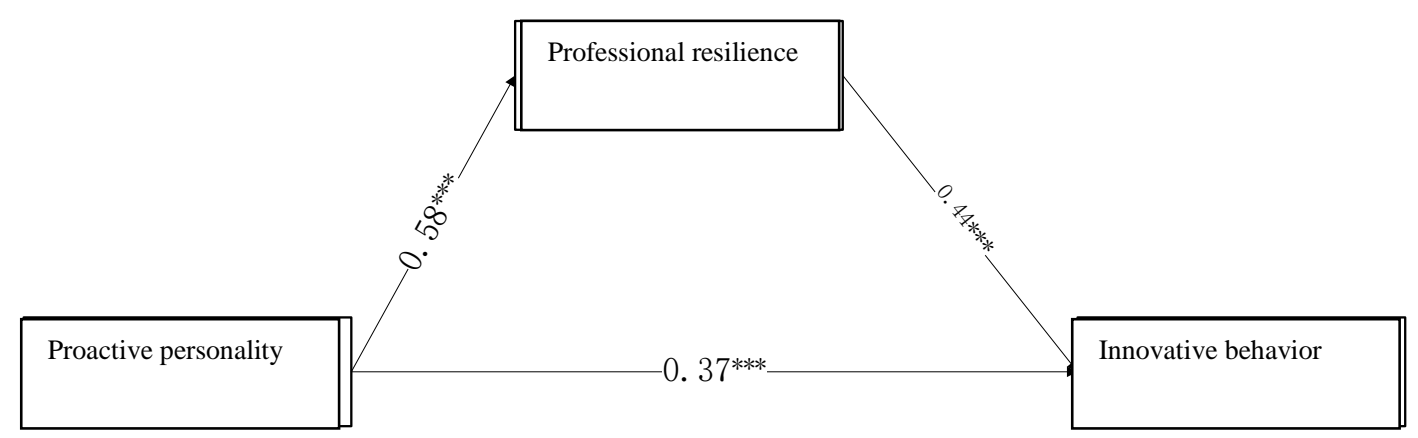

Figure 2 The effect of proactive personality on innovation behavior.

\section{DISCUSSIONS}

The research on the innovative behavior of military civil personnel has always been the focus and problem that the military human resource management department pays attention to, and it is also the starting point and goal of the military human resource system reform. Through empirical analysis, this research confirms the proactive personality and the influence on innovative behavior and internal mechanism, and uncovers the dark box of civil personnel's innovative behavior, which has high value in theory and practice.

\subsection{The Impact of the Proactive Personality of Civil Personnel on Innovative Behavior}

Studies have shown that the proactive personality of civil personnel has a significant positive impact on innovative behavior. This conclusion is consistent with the research conclusions of Zhou Yufan [16] and Zhang Yamei [17]. The possible reason is that the typical feature of proactive personality is that it is not bound to the external environment and can bring about changes in the surrounding environment [12]. As a stable and long-lasting personality trait, it is more likely to have a long-term and profound impact on organizational behavior. Individuals with high proactive personality are more inclined to use active behaviors to influence and change the surrounding environment and things, can recognize and grasp the opportunities around them in time, are willing to make long-term efforts to achieve goals, and have stronger behavioral autonomy. As an organization behavior outside of roles, innovation needs to be supported by a high degree of autonomy and long-term efforts rather than passive execution. In addition, based on the perspective of resource conservation theory, individuals with proactive personality are more likely to obtain superior resources in their work, and these resources will also promote the creation of innovative behaviors to a certain extent. Based on this, proactive personality has a significant role in promoting innovative behavior.

\subsection{The Mediating Role of Professional Resilience Between the Proactive Personality and Innovative Behavior of Civil Personnel}

The study also found that, in addition to directly affecting the creative behavior of civil personnel, professional resilience can also indirectly affect innovation behavior through the "bridge" function of it. This conclusion further reveals the internal mechanism of proactive personality influencing innovation behavior. At the same time, it also takes civil personnel as the research object to enrich and expand the external validity of the conclusion. The reason why proactive personality affects professional resilience may have such a logical relationship. For individuals with high initiative personality, when faced with challenging tasks and work, they often not shrink back, but show more confidence, have stronger willpower, and be able to work hard for the goals they set. Even when they are in adversity or troubled by problems, they can still be good at finding opportunities and achieving success [18]. Some researchers even pointed out that in the Chinese cultural background, the proactive personality should contain the component of resilience [19]. From another perspective, even when encountering setbacks and innovation failures at work, they are often able to use high-quality and efficient social support networks and various resources to properly solve these difficulties and show strong professional resilience. Conclusions are also reflected in previous studies [20]. The role of professional resilience in promoting innovative behavior is reflected in most studies. For example, Ren Yunxia's research has shown that professional 
resilience has a significant positive impact on innovative behaviors, and it acts as an intermediary between the support of others and innovative behaviors[11]. Li Meifang's research shows that resilience in psychological capital is positively correlated with innovative behavior[21]. When individuals engage in innovative behaviors, they will inevitably encounter various difficulties and challenges. Innovation failures are inevitable and require strong professional resilience as support. For individuals with low professional resilience, facing innovation failures, they are likely to fail and lose their motivation to continue to innovate. Only individuals with high professional resilience can maintain high-frequency innovative behaviors until they succeed. It can be seen that the proactive personality of civil personnel will influence innovative behavior through the mediating effect of professional resilience.

\subsection{The Enlightenment of Research Conclusions on Civil Personnel Management and Research Limitations}

The research conclusions of this article have certain enlightenment for the management of civilian personnel, and also provide some reference for how to stimulate the innovative behavior of civil personnel. At least, from the results of this article, there are two measures that can be adopted. First of all, it is necessary to make efforts in the recruitment of civil personnel. Under the same conditions, priority will be given to recruiting candidates with stronger initiative and personality to supplement the civil personnel. For the civil personnel that have been hired, specific education and training forms can be used to shape their proactive personality. Second, it is also necessary to pay full attention to the professional resilience of civil personnel. For civil personnel who have just entered the workplace, they have little and insufficient experience, and a low level of professional resilience. Their professional resilience can be improved through psychological behavior training or frustration education, so that they can make timely adjustments in the face of innovation failure and invest in innovation again.

However, this study also has certain limitations. For example, the data used in the research are all cross-sectional data. Although there is sufficient theoretical support, it is not convincing in revealing the causal relationship. Future research can adopt the method of tracking research to more accurately reveal the causal relationship between variables. In addition, as a complex organizational behavior, innovation behavior may involve more complex mechanisms of action. This research is only the tip of the iceberg. Future research can explore more variables and more complex mechanisms of action.

\section{CONCLUSION}

The proactive personality of military civil personnel has a significant positive predictive effect on innovative behavior, that is, the stronger the proactive personality, the stronger the innovative behavior.

The proactive personality of military civil personnel can not only directly affect innovation behavior, but also can affect innovation behavior through the indirect effect of professional resilience, that is, professional resilience plays a part of mediating role between proactive personality and innovative behavior.

\section{AUTHORS' CONTRIBUTIONS}

Yanhui Tian wrote the paper, Ting Wei and Zhengyin Wang is responsible for research design,Fei Liu and Hui Liu analysed the data.

\section{REFERENCES}

[1] Janssen O, Vliert E V D, West M. The Bright and Dark Sides of Individual and Group Innovation: A Special Issue Introduction[J]. Journal of Organizational Behavior, 2004, 25(2): 129-145.

[2] Li M, Hsu C H C. A Review of Employee Innovative Behavior in Services [J]. International Journal of Contemporary Hospitality Management, 2016, 28(12): 28202841.

[3] Montani F, Courcy F O, Vandenberghe C. Innovating Under Stress: The Role of Commitment and Leader-member Exchange [J]. Journal of Business Research, 2017, 77: 113.

[4] Zhang Suchuan, Chen Lixin, The Impact of an Inclusive Atmosphere on Employee Innovation Behavior Based on Data Surveys in Shanxi and Inner Mongolia [J]. Journal of Chongqing Technology and Business University (Social Science Edition), 2020: 114. (in Chinese) 
[5] Zhou Jinbo, Song Zhanyang, Influencing Mechanism of Leadership Empowerment on Employees' Innovative Behavior: The Regulating Role of Uncertainty Avoidance and Error Management Atmosphere [J]. Science and Technology Management Research, 2020, 40(14): 140-148. (in Chinese)

[6] Ding Daoren, Effects of Perceived Organizational Status on Innovative Proactive Behavior Based on the Mediating Effect of Psychological Capital and Moderating Effect of Transformational Leadership [J]. Journal of Systems \& Management, 2020, 29(02): 326334. (in Chinese)

[7] Gao Peng, Xue Pu, Xie Ying, The Influence of Self-Monitoring Personality on Innovation Performance: Based on the Theory of Social Information Processing $[\mathrm{J}]$. Science \& Technology Progress and Policy, 2020: 1-10. (in Chinese)

[8] Ma Wei, Su Hang, The Influence of the Perceived Climate of Team Cha-Xu on Employee Innovation Behavior [J]. Science \& Technology Progress and Policy, 2020, 37(21):136-143. (in Chinese)

[9] Zhang Ying, Yang Fu, Proactive Personality: Mechanisms and Future Directions [J]. Advances in Psychological Science, 2017, 25(09): 1544-1551. (in Chinese)

[10] S C J M B. Charismatic Leadership Viewed from above: The Impact of Proactive Personality [Z]. 2000: 21, 63-75.

[11] Ren Yunxia, On the Influence Mechanism of Support from Important Others on the Innovative Behaviors of New Generation Employees:Based on the Roles of Career Resilience and Corporate Culture [J]. Journal of China University of Petroleum (Edition of Social Sciences), 2019, 35(05): 33-39. (in Chinese)

[12] Bateman T S, M C J. The proactive component of organizational behavior. A measure and correlates [Z]. 1993: 14, 103118.

[13] London M N R. London's career motivation theory:Anupdate on measurement and research[Z]. 1997: 5, 61-80.

[14] Scott S G, Bruce R A. Determinants of Innovative Behavior A Path Model of
Individual Innovation in the Workplace [J]. The Academy of Management Journal, 1994, 37(3):1442-1465.

[15] AF H. Introduction to Mediation, Moderation, and Conditional Process Analysis: A Regression-Based Approach[Z]. New York: Guilford Press, 2013.

[16] Zhou Yufan, Zhang Jianwei, Zhang Chenyu, etc. The Impact of Proactive Personality on R\&O Personnel Innovative Behavior - An Integrated Perspective of Trait Activation Theory and Conservation of Resources Theory [J]. Soft Science, 2020, 34(07): 33-37. (in Chinese)

[17] Zhang Yamei, A Study on the Relationship Between Internal Organizational Environment, Proactive Personality and the Sustainability of Entrepreneurial Innovation Behavior [D]. Yunnan University of Finance and Economics, 2020. (in Chinese)

[18] Shi Bianmei, The Effect of Proactive Personality on College Students' Creativity: The Mediation Role of Psychological Capital and Creative Motivation [D]. Zhejiang University, 2014. (in Chinese)

[19] Ye Lianhua, Ling Wenquan, Proactive Personality in I/O Psychology [J]. Advances in Psychological Science, 2007(03): 498-504. (in Chinese)

[20] Yan Wei, The Influence of Corporate Employees' Initiative Personality and Psychological Capital on Organizational Citizenship Behavior and Anti-productive Behavior [D]. Harbin Normal University, 2010. (in Chinese)

[21] Li Meifang, Research on the Relationship between Psychological Capital and Innovative Behavior of Corporation's Knowledge Worker [D]. Shandong University, 2011. (in Chinese) 